\title{
Comparison of the Accuracy of 3D Printed Prototypes Using the Stereolithography (SLA) Method with the Digital CAD Models
}

\author{
Emil Yankov ${ }^{1, *}$ and Maria P. Nikolova ${ }^{1}$ \\ ${ }^{1}$ University of Ruse, Dept. Material Science and Technology, Ruse, 8 Studentska Str., Bulgaria
}

\begin{abstract}
Each printing method and the materials used for prototype construction have distinct advantages and drawbacks. A large part of the 3D printer producers indicates high printing accuracy (positioning accuracies from $100 \mu \mathrm{m}$ up to $1 \mu \mathrm{m}$ and layer thickness from $100 \mu \mathrm{m}$ to 5 $\mu \mathrm{m})$. In the process of layers deposition, the material is softened, melted or irradiated with a certain light source to polymerize. Thus, during the printing process, the volume of the materials is changing causing shrinking or expansion which often leads to the occurrence of precision errors in the prototype as opposed to the digital model. This phenomenon leads to distortions of the printed object known as a curling effect. Certain chemical compounds are added by the producers to multiple materials in order to enhance their mechanical and technological properties. However, this often results in changes of some printing parameters influencing the precision and quality of the manufactured object. The aim of the particular study is to establish the allowances with regard to the accuracy during manufacturing precise objects used in micro technologies as well as the necessary adjustments in dimensions because of the shrinkage and the positioning of the SLA 3D printed parts from photopolymer material.
\end{abstract}

\section{Introduction}

In recent years the development of electronics, machine building and micro-technologies has imposed extreme progress of different kinds of 3D printers (FDM, SLS, SLA, SLM, DLP, DMLS, EBM, etc.) working with wide variation of materials (PLA, ABS, PETG, PVA, photopolymers, ceramics, metals, etc.). The stereolithography is an additive process of $3 \mathrm{D}$ printing with liquid photopolymer that polymerizes under the laser irradiation. The work table is moving up- and downwards and after immersing in the resin the photopolymer hardens by selective irradiation of the laser beam. In that way, the printed object is formed as a bottom-up physical model with clearly discernible details.

In recent years 3D models of various anatomical regions have been presented in the international "3D Printing for professional - London 2016". The scientists from Washington State University are printing bone-like structures („Inspiring ingenuity Printing new bone") that could be used in orthopedic and dental procedures $[1,2]$. This

\footnotetext{
*Corresponding author: eyankov@uni-ruse.bg
} 
bone-like material is acting as a scaffold for the new bone growth and after that, it dissolves with no side effects or needs for surgical interventions. Additionally, the complex anatomical shapes of the craniofacial or ear prosthesis, for example, are hard to be manufactured by conventional methods. The 3D printing can cope with this challenges of today more quickly and at less cost. After scanning the object with 3D scanners or cameras "https://digitalscan3d.com/" and thus generating the high-quality mesh, the digital model for the 3D printing is acquired. Simultaneously, the 3D solid model accuracy depends on the physical properties of the material during heating and cooling that results in changes of the object dimensions. With the increase of the model size, the errors accumulate for each single layer. These errors show positive or negative absolute value that could affect the durability and exploitation of the object afterward. The ultraviolet oven treatment changed also the solid model dimensions because of the residual stresses locked in between the layered structure. That's why the aim of the particular study is to establish the allowances with regard to the accuracy during SLA 3D printing as well as the necessary adjustments in dimensions because of the shrinkage and the positioning of the printed parts from photopolymer material.

\section{Experimental procedures}

The digital model was constructed by CAD system SolidWorks. The size of the model was $30 \times 30 \mathrm{~mm}$ and $3 \mathrm{~mm}$ thickness. The micro-squares within the model had dimensions of $200 \times 200 \mu \mathrm{m}$ and depth of $500 \mu \mathrm{m}$ with a separating interval of $400 \mu \mathrm{m}$ in $\mathrm{X}$ and $\mathrm{Y}$ directions (Fig. 1 a). The printed model was received by means of SLA printer Formlabs 2.0 (Fig. 1 b) using standard photopolymer "Clear" and $25 \mu \mathrm{m}$ prescribed values of printing precision. The model was $45^{\circ}$ tilted with respect to $\mathrm{X}, \mathrm{Y}$ and $\mathrm{Z}$ axis in the work space and the supporting props were fitted at the edges of the object in order not to affect the examined area. For ensuring the fixation stability the distance between the props was selected to be $600 \mu \mathrm{m}$ and the size of fastening point between the model and the supporting prop was $400 \mu \mathrm{m}$. The supporting props were reinforced by a belt with $3.5 \mathrm{~mm}$ thickness positioned in bedplate of the model. The depth of the base sticking to the work table was equal to $1 \mathrm{~mm}$.

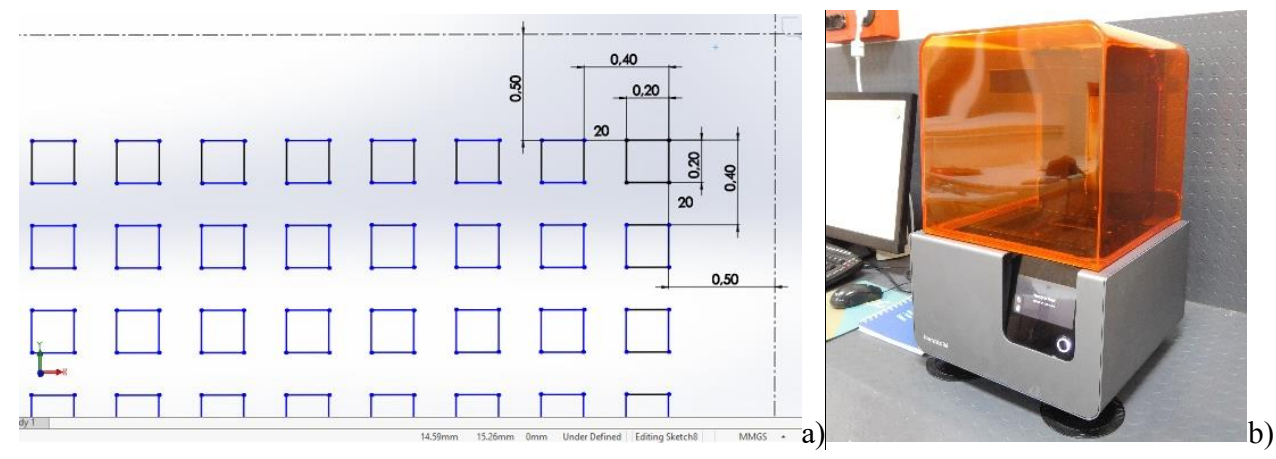

Fig. 1. Manufacturing of physical 3D model: a) providing of the net of micro-squares; b) Formlabs 2 3D printer.

The measurement of the coordinates of the micro-squares after the $3 \mathrm{D}$ printing was carried out by a "Carl Zeiss" optical microscope at $12.5 \times$ magnification. The digital micrograph acquired by 5 Mpix camera and S-EYE software (Fig. 2) was used to measure the micro-grid deviations of the printed model. 
As shown in Fig. 3, the coordinate grid method [3-5] that consists of measuring the relative "distortions" in different directions $\left(\varepsilon_{\mathrm{x}}\right.$ and $\left.\varepsilon_{\mathrm{y}}\right)$ was used to calculate the deviations during the photopolymerization of the printed model. The following equations were used:

$$
\varepsilon_{X}=\frac{\Delta X}{X_{i}}, \varepsilon_{Y}=\frac{\Delta Y}{Y_{i}}
$$

Where $\Delta \mathrm{X}$ was the drift value of the $\mathrm{X}$ axis and $\Delta \mathrm{Y}$ was the drift value of the $\mathrm{Y}$ axis. The $\mathrm{X}_{i}$ and $\mathrm{Y}_{i}$ were the original (initial) dimensions in the model and $\mathrm{X}_{n, i}$ and $\mathrm{Y}_{n, i}$ was the changed values in dimension (Fig. 3).

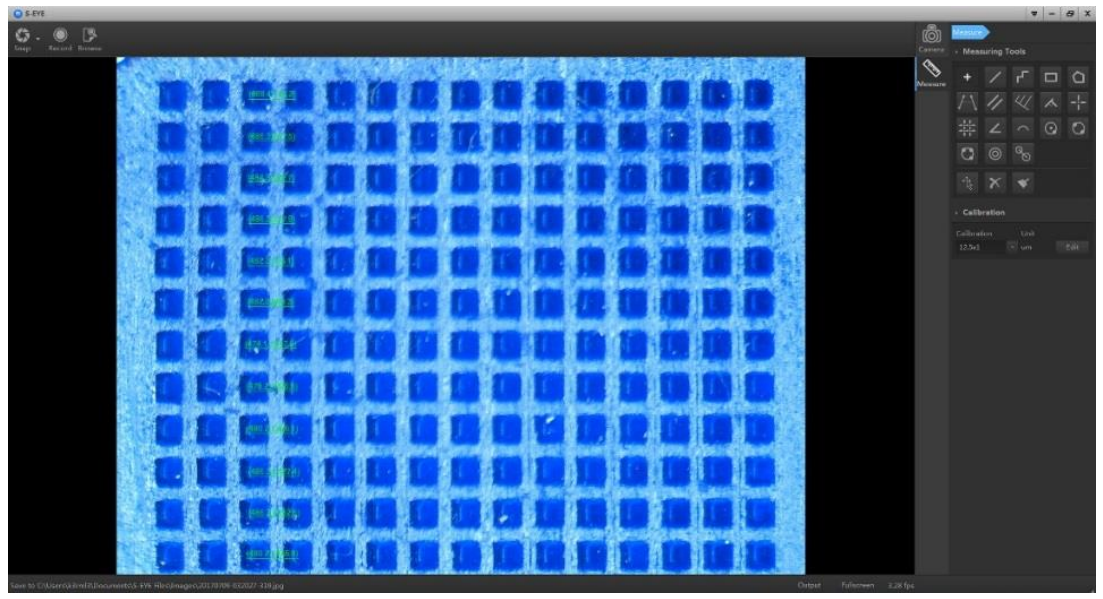

Fig. 2. Digital image and measured values of the $3 \mathrm{D}$ printed model.

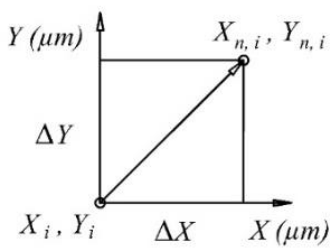

Fig. 3. The setting of the deviations by the coordinate grid method.

The coordinates of one of the corner point were used as reference fiducial marks. These values were entered into Excel tables in order to determine the differences between the coordinates of the specified digital model and those of the 3D printed prototype. The measured $\mathrm{X}$ and $\mathrm{Y}$ values were split into two separate tables for the purpose of defining appropriate tolerances between the prototype and the digital model of both axes.

\section{Experimental results}

After the mathematical processing of the results obtained (Fig. 4), the data are presented in graphical form (Fig. 5) showing the relative deviations of each cell against the reference digital model. Fig. 4 shows the tabular deviation in the values by color code in $\mathrm{X}$ and $\mathrm{Y}$ directions. A positive displacement could be noted in the upper left part of the model. That is probably a consequence of the chosen option of positioning and the selected fixation scheme. A further reason for this effect could be the model hanging and the near located supporting props. 


\begin{tabular}{|c|c|c|c|c|c|c|c|c|c|c|c|c|c|c|c|}
\hline & 1 & 2 & 3 & 4 & 5 & 6 & 7 & 8 & 9 & 10 & 11 & 12 & 13 & 14 & 15 \\
\hline 1 & 0.550 & -2.135 & -1.460 & -3.795 & -3.460 & -5.485 & -5.460 & -7.165 & -7.460 & -8.855 & -9.470 & -10.525 & -11.460 & -12.225 & -13.460 \\
\hline 2 & -1.130 & -2.145 & -3.150 & -3.795 & -5.150 & -5.475 & -7.140 & -7.175 & -9.130 & -8.845 & -11.150 & -10.535 & -13.150 & -12.215 & -15.140 \\
\hline 3 & -2.830 & -2.115 & -4.830 & -3.785 & -6.830 & -5.485 & -8.830 & -7.175 & -10.830 & -8.835 & -12.830 & -10.535 & -14.850 & -12.205 & -16.830 \\
\hline 4 & -4.520 & -2.115 & -6.510 & -3.805 & -8.530 & -5.455 & -10.510 & -7.155 & -12.520 & -8.865 & -14.500 & -10.545 & -16.520 & -12.235 & -18.520 \\
\hline 5 & -6.210 & -2.125 & -8.210 & -3.795 & -10.210 & -5.485 & -12.210 & -7.155 & -14.190 & -8.865 & -16.190 & -10.515 & -18.200 & -12.205 & -20.200 \\
\hline 6 & -7.900 & -2.105 & -9.890 & -3.785 & -11.900 & -5.485 & -13.910 & -7.165 & -15.900 & -8.850 & -17.860 & -10.535 & -19.880 & -12.215 & -21.870 \\
\hline 7 & -9.580 & -2.105 & -11.600 & -3.785 & -13.570 & -5.465 & -15.570 & -7.175 & -17.580 & -8.865 & -19.560 & -10.525 & -21.580 & -12.225 & -23.580 \\
\hline 8 & -11.270 & -2.115 & -13.260 & -3.785 & -15.260 & -5.485 & -17.250 & -7.165 & -19.260 & -8.845 & -21.280 & -10.525 & -23.260 & -12.205 & -25.270 \\
\hline 9 & -12.940 & -2.125 & -14.950 & -3.795 & -16.940 & -5.495 & -18.950 & -7.185 & -20.930 & -8.855 & -22.950 & -10.515 & -24.930 & -12.215 & -26.930 \\
\hline 10 & -14.630 & -2.115 & -16.620 & -3.775 & -18.620 & -5.475 & -20.640 & -7.155 & -22.630 & -8.855 & -24.630 & -10.535 & -26.650 & -12.195 & -28.630 \\
\hline 11 & -16.320 & -2.105 & -18.320 & -3.775 & -20.310 & -5.485 & -22.320 & -7.185 & -24.320 & -8.850 & -26.320 & -10.515 & -28.310 & -12.225 & -30.320 \\
\hline 12 & -18.000 & -2.095 & -19.990 & -3.795 & -22.000 & -5.485 & -24.000 & -7.165 & -26.000 & -8.855 & -28.000 & -10.535 & -29.990 & -12.235 & -32.000 \\
\hline 13 & -19.680 & -2.125 & -21.700 & -3.785 & -23.680 & -5.475 & -25.690 & -7.175 & -27.680 & -8.835 & -29.670 & -10.545 & -31.690 & -12.215 & -33.700 \\
\hline 14 & -21.400 & -2.095 & -23.370 & -3.805 & -25.380 & -5.485 & -27.370 & -7.175 & -29.370 & -8.865 & -31.370 & -10.535 & -33.370 & -12.205 & -35.370 \\
\hline 15 & -23.050 & -2.125 & -25.080 & -3.795 & -27.060 & -5.495 & -29.040 & -7.155 & -31.050 & -8.835 & -33.040 & -10.535 & -35.060 & -12.225 & -37.060 \\
\hline
\end{tabular}

\begin{tabular}{|c|c|c|c|c|c|c|c|c|c|c|c|c|c|c|c|c|}
\hline & 1 & 2 & 3 & 4 & 5 & 6 & 7 & 8 & 9 & 10 & 11 & 12 & 13 & 14 & 15 & \\
\hline 1 & -0.425 & 0.550 & -2.135 & -1.460 & -3.795 & -3.460 & -5.485 & -5.460 & -7.165 & -7.460 & -8.855 & -9.470 & -10.525 & -11.460 & -12.225 & 1 \\
\hline 2 & -0.405 & -1.130 & -2.145 & -3.150 & -3.795 & -5.150 & -5.475 & -7.140 & -7.175 & -9.130 & -8.845 & -11.150 & -10.535 & -13.150 & -12.215 & 2 \\
\hline 3 & -0.435 & -2.830 & -2.115 & -4.830 & -3.785 & -6.830 & -5.485 & -8.830 & -7.175 & -10.830 & -8.835 & -12.830 & -10.535 & -14.850 & -12.205 & 3 \\
\hline 4 & -0.415 & -4.520 & -2.115 & -6.510 & -3.805 & -8.530 & -5.455 & -10.510 & -7.155 & -12.520 & -8.865 & -14.500 & -10.545 & -16.520 & -12.235 & 4 \\
\hline 5 & -0.455 & -6.210 & -2.125 & -8.210 & -3.795 & -10.210 & -5.485 & -12.210 & -7.155 & -14.190 & -8.865 & -16.190 & -10.515 & -18.200 & -12.205 & 5 \\
\hline 6 & -0.425 & -7.900 & -2.105 & -9.890 & -3.785 & -11.900 & -5.485 & -13.910 & -7.165 & -15.900 & -8.850 & -17.860 & -10.535 & -19.880 & -12.215 & 6 \\
\hline 7 & -0.415 & -9.580 & -2.105 & -11.600 & -3.785 & -13.570 & -5.465 & -15.570 & -7.175 & -17.580 & -8.865 & -19.560 & -10.525 & -21.580 & -12.225 & 7 \\
\hline 8 & -0.425 & -11.270 & -2.115 & -13.260 & -3.785 & -15.260 & -5.485 & -17.250 & -7.165 & -19.260 & -8.845 & -21.280 & -10.525 & -23.260 & -12.205 & 8 \\
\hline 9 & -0.425 & -12.940 & -2.125 & -14.950 & -3.795 & -16.940 & -5.495 & -18.950 & -7.185 & -20.930 & -8.855 & -22.950 & -10.515 & -24.930 & -12.215 & 9 \\
\hline 10 & -0.425 & -14.630 & -2.115 & -16.620 & -3.775 & -18.620 & -5.475 & -20.640 & -7.155 & -22.630 & -8.855 & -24.630 & -10.535 & -26.650 & -12.195 & 10 \\
\hline 11 & -0.435 & -16.320 & -2.105 & -18.320 & -3.775 & -20.310 & -5.485 & -22.320 & -7.185 & -24.320 & -8.850 & -26.320 & -10.515 & -28.310 & -12.225 & 11 \\
\hline 12 & -0.425 & -18.000 & -2.095 & -19.990 & -3.795 & -22.000 & -5.485 & -24.000 & -7.165 & -26.000 & -8.855 & -28.000 & -10.535 & -29.990 & -12.235 & 12 \\
\hline 13 & -0.415 & -19.680 & -2.125 & -21.700 & -3.785 & -23.680 & -5.475 & -25.690 & -7.175 & -27.680 & -8.835 & -29.670 & -10.545 & -31.690 & -12.215 & 13 \\
\hline 14 & -0.425 & -21.400 & -2.095 & -23.370 & -3.805 & -25.380 & -5.485 & -27.370 & -7.175 & -29.370 & -8.865 & -31.370 & -10.535 & -33.370 & -12.205 & 14 \\
\hline 15 & -0.415 & -23.050 & -2.125 & -25.080 & -3.795 & -27.060 & -5.495 & -29.040 & -7.155 & -31.050 & -8.835 & -33.040 & -10.535 & -35.060 & -12.225 & 15 \\
\hline
\end{tabular}

Fig. 4. Colour relative deviation of the coordinates of: a) $\mathrm{X}$ axis; b) $\mathrm{Y}$ axis. 
In contrast, in the lower right corner, there is a size reduction of the model. The reason for this could be the model shrinking after exposure to the UV lamp and the additional heat setting.

The calculated relative values are presented in 3D graphical form (Fig. 5) with the aim of obtaining a dimensional picture for the tolerances in $\mathrm{X}$ and $\mathrm{Y}$ directions after the 3D printing.

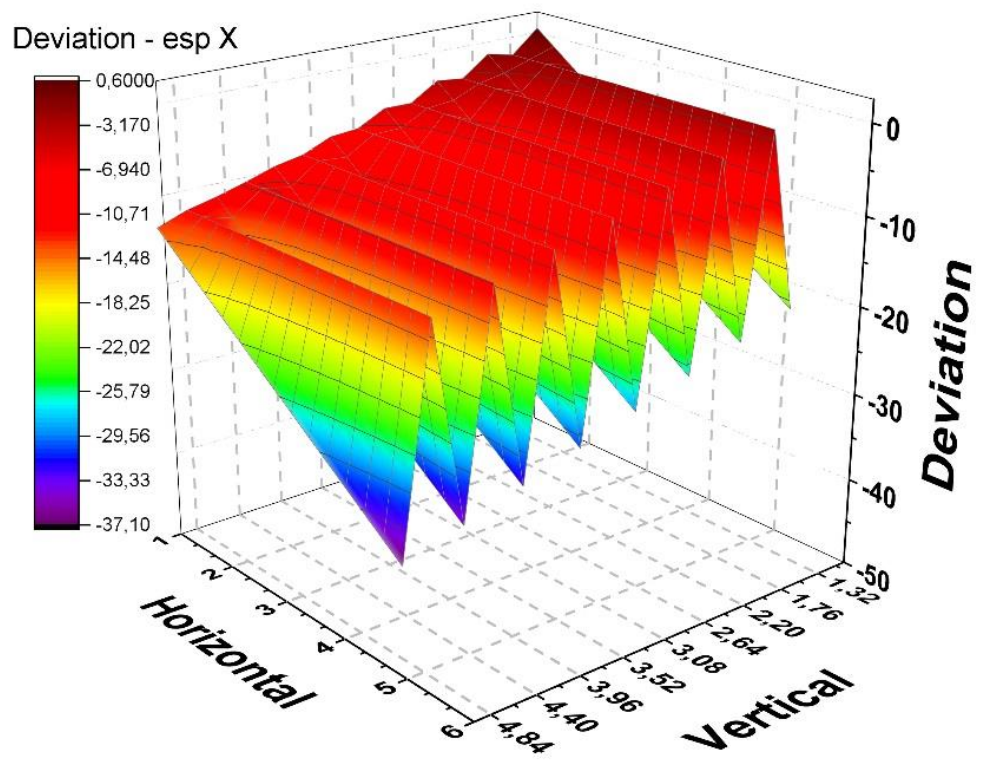

a)

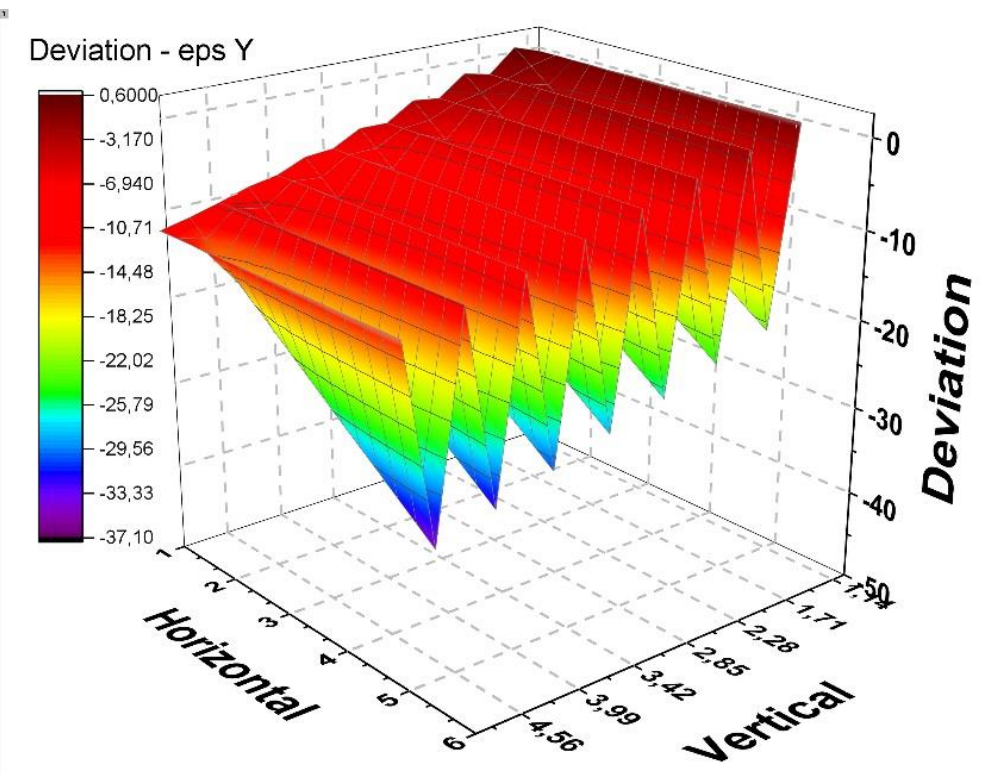

b)

Fig. 5. 3D graph of the size deviation of: a) $\mathrm{X}$ axis; b) $\mathrm{Y}$ axis. 
The deviations in both $\mathrm{X}$ and $\mathrm{Y}$ axis increase with the rise in the number of layers (Fig. 5). These errors accumulate and show permanent character shifting towards the negative values during the building up of the solid model.

\section{Conclusions}

The main conclusions drawn on the basis of the particular research are:

- The coordinate grid method allows rapid and efficient measurement of the deviations in the nominal size values of the printed (micro) parts. Additionally, the changes in size could also be determined in different directions (for example $\mathrm{X}$ and $\mathrm{Y}$ ).

- The lowest divergences in the nominal size values are measured in the upper part of the printed model where the first layer is built and the model is near to the moving table. This solid part of the object is connected to the table with shorter props. This means that the random and systematic deviations in the $\mathrm{X}$ direction (parallel to the table surface) are less. - An additional factor to consider is the immersion and removal of the part. The more the built layers are moving away from the table, the more their "mobility" will grow and the deviations increase. The highest is the size deviations in the last polymerized layers.

- With regard to the $\mathrm{Y}$ axis deviations - $\varepsilon_{\mathrm{y}}$ (eps $\mathrm{Y}$ ), the same trend is seen: the greater the distance from the table, the higher and more irregular the deviations with respect to the absolute values of the digital model.

- In the upper part of the object near to the table, the dispersion of the values is similar with respect to the end of the solid model but the latter are shifted towards negative values at both $\mathrm{X}$ and $\mathrm{Y}$ axis.

- The results obtained will be valuable for introducing of size corrections in the digital models in order to print more accurate $3 \mathrm{D}$ physical model.

In order to determine the reasons and confirm the results, additional examinations will be needed.

\section{References}

1. H. Y. Yu, D. D. Ma, B. L. Wu, Nan Fang Yi Ke Da Xue Xue Bao (Journal of Southern Medical University), 37 (5) 668-672 (2017)

2. M. A. Kuss, R. Harms, S. Wu, Y. Wang, J. B. Untrauer, M. A. Carlson, B. Duan, RSC Advances 7, 29312-29320 (2017)

3. N. Feschiev, V. Gagov, E. Minev, Conf. Proc. Avangard technologies and machine building materials, VMEI Gabrovo, 187-191 (1992)

4. B. Tomov, V. Gagov, N. Feschiev, E. Minev, Proc. of $9^{\text {th }}$ Machine Tool Conference, University Politechnica of Bucharest, 9 264-269 (1994)

5. V. Gagov, Testing and Processing of Contemporary Materials, Ljubljana-RuseGliwice, 125-136 (1997) 